journal club

\title{
Antikoagulation bei Vorhofflimmern im Praxisalltag - Metaanalyse von Registerdaten
}

Fragestellung: Wie wirksam sind Rivaroxaban und Dabigatran verglichen mit Warfarin bei der Schlaganfallprävention bei Vorhofflimmern in der klinischen Praxis?

Hintergrund: Vorhofflimmern ist ein wichtiger Risikofaktor für den ischämischen Schlaganfall. Durch Vitamin-K-Antagonisten (VKA) wie Warfarin kann das Schlaganfallrisiko um 60-70\% im Vergleich zu Placebo reduziert werden. Die Nicht-Vitamin-K-abhängigen oralen Antikoagulanzien (NOAK) Rivaroxaban und Dabigatran waren in randomisierten Studien entweder vergleichbar wirksam wie Warfarin oder überlegen bezüglich der Schlaganfallprävention. Beide Substanzen führten in randomisierten Studien wie ROCKET-AF und RE-LY zu einer signifikanten Reduktion intrakanieller Blutungen. In der Zwischenzeit gibt es eine Vielzahl von Registerdaten, die die Anwendung der beiden Antikoagulanzen in der klinischen Praxis widerspiegeln. Diese sollten jetzt in einer Metaanalyse ausgewertet werden.

Bai Y, Deng H, Shantsila A et al. Rivaroxaban versus dabigatran or warfarin in real-world studies of stroke prevention in atrial fibrillation: Systematic review and meta-analysis. Stroke 2017; doi: 10.1161/STROKEAHA.116.016275
Patienten und Methodik: In die Metaanalysen wurden 17 Registerstudien eingeschlossen, in denen die Wirksamkeit und die Sicherheit von Rivaroxaban versus Dabigatran (3 Studien), Rivaroxaban versus Warfarin (11 Studien) oder Rivaroxaban ver- sus Dabigatran versus Warfarin (3 Studien) für die Schlaganfallprävention bei Vorhofflimmern verglichen wurden.

Ergebnisse: Für den Endpunkt Schlaganfall war bezüglich der Wirksamkeit zwischen Rivaroxaban und Dabigatran kein Unterschied festzustellen. Die Hazard Ratio [HR] für schwerwiegende Blutungskomplikationen war für Rivaroxaban verglichen mit Dabigatran 1,38. Dies bedeutet eine signifikante Risikoerhöhung zuungunsten von Rivaroxaban. Beim Vergleich zwischen Rivaroxaban und Warfarin bezüglich Schlaganfall betrug die HR 0,75 zugunsten von Rivaroxaban. Dies bedeutete eine signifikante Überlegenheit von Rivaroxaban im Vergleich zu Warfarin. Im Vergleich bezüglich schwerwiegender Blutungskomplikationen bestand zwischen Warfarin und Rivaroxaban kein Unterschied. Wurden die Blutungen unterteilt, ergab sich für Rivaroxaban versus Warfarin ein erhöhtes Risiko gastrointestinaler Blutungen und ein reduziertes Risiko intrakranieller Blutungen.

Schlussfolgerungen: In einer Metaanalyse von Registerdaten waren Rivaroxaban und Dabigatran bezüglich der Schlaganfallprävention vergleichbar gut wirksam. Bezüglich dieses Endpunkts war Rivaroxaban Warfarin überlegen. Bei den schwerwiegenden Blutungskomplikationen war Dabigatran Rivaroxaban überlegen. Im Vergleich von Rivaroxaban und Warfarin war die Gesamtblutungsrate vergleichbar, das Risiko gastrointestinaler Blutungen unter Rivaroxaban versus Warfarin erhöht und das Risiko intrakranieller Blutungen für beide Substanzen reduziert.

\section{- Kommentar von Hans-Christoph Diener, Essen}

\section{Ein direkter Vergleich ist nur gegenüber Warfarin möglich}

Ein direkter Vergleich der Ergebnisse der randomisierten Studien zu Rivaroxaban und Dabigatran versus Warfarin ist nicht möglich, da die beiden Studien ROCKET-AF und RE-LY unterschiedliche Patientenpopulationen einschlossen. So hatten bereits $50 \%$ der Patienten in der ROCKET-AF Studie eine TIA oder einen Schlaganfall erlitten und damit ein deutlich höheres Rezidivrisiko als die Patienten, die in RE-LY eingeschlossen wurden. Registerdaten erlauben allerdings im begrenzten Umfang einen direkten Vergleich zwischen den beiden NOAK Rivaroxaban und Dabigatran gegenüber Warfarin. Bezüglich des primären Ziels der Schlaganfallprävention, nämlich Schlaganfälle zu verhindern, waren Rivaroxaban und Dabigatran gleich wirksam wie Warfarin, wobei nicht berücksichtigt wurde, welche Dosis von Dabigatran gegeben wurde. Rivaroxaban hat sowohl im Vergleich zu Dabigatran wie im Vergleich zu Warfarin ein erhöhtes Blutungsrisiko. Dies ist aber überwiegend durch das Risiko gastrointestinaler Blutungen bedingt, die relativ gut behandelt werden können. Die Blutungskom- plikation, die mit der höchsten Sterblichkeit einhergeht, nämlich intrakranielle Blutungen, waren unter Rivaroxaban signifikant seltener als unter Warfarin. Dies spiegelt das Ergebnis der randomisierten ROCKET-AF-Studie wider.

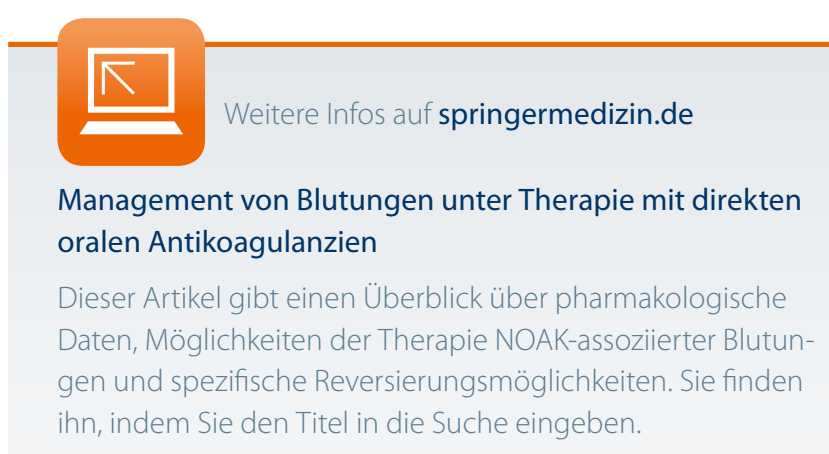

